\author{
Giandomenico Passavanti ${ }^{1 *}$ and Anna \\ Maria Aloisi ${ }^{2}$ \\ ${ }^{1}$ Departement of Urology "Misericordia Hospital" \\ GROSSETO, Italy \\ ${ }^{2}$ Departement of Physisology University of SIENA, \\ Italy \\ Dates: Received: 19 August, 2015; Accepted: 19 \\ October, 2015; Published: 21 October, 2015 \\ *Corresponding author: Giandomenico Passavanti, \\ Departement of Urology Misericordia Hospital \\ GROSSETO -Italy, Via Oberdan, 4458100 \\ GROSSETO, Italy, Tel: +39 (0)564411864; Fax: +39 \\ (0)564411864; E-mail: rosapass@libero.it \\ www.peertechz.com \\ ISSN: 2455-5495
}

\section{Research Article \\ Surgery in Peyronie's Disease: Our Experiences with Tunica Plication, Plaque Incision and Grafting with Gore-Tex, and Satisfaction Degree of the Patients}

\section{Introduction}

The Peyronye's disease is a frequent condition and it causes a penile deformity which causes coital dysfunction and psychological disorders because of the difficulty in coping with the situation.

It is important to carry out a thorough diagnostic study of patients to propose the proper treatment; furthermore it is necessary to understand patient expectations and inform them about the treatment and about the results which the therapy can offers.
Through our experiences we understood some relevant aspects of this disease and its therapy, which we are showing in this study.

Table 1: Auto-evaluation of surgical satisfaction Score.

1 unsatisfied

2 satisfied

3 enough satisfied

4 a lot satisfied 


\section{Materials and Methods}

46 patients underwent surgery for recurvatum penis secondary to Peyronie's disease; patients age was between 51 and 76 years (m. 67,8 years).

All patients have had a stable disease for about 1year at the time of surgery. They reported severe coital dysfunction associated to psychological discomfort to the erectile penile deformity.

All patients had a thorough clinical history and examination to identify the plaques.

We carried out an ultrasonography to identify the cases with calcifications (4 cases).

The erectile function was examined with IIEF5 and in case of erectile dysfunction we performed intracavernous injection of PGE1.

The photography of penis during erection documented the recurvatum degree (Figure 1) and sandglass deformity. Penis length measured both during flaccidity and with stretching. We used a rigid ruler and measured the penis from pubis to glans.

40 patients had a moderate recurvatum $\left(45-60^{\circ}\right)$ with difficult penetration. The erectile function was preserved in 31 cases and 9 had ED well treated with 5PDEi.

Penile length prior plication surgery was between 12 and 16,5 $\mathrm{cm}(\mathrm{m} .14+/-1,2 \mathrm{~cm})$. In patients with ED plasma testosterone was measured. Sandglass deformity was evident in 20 patients, with all patients coping well with it.

40 patients with moderate recurvatum underwent surgery with albuginea plication; 14 patients had albuginea stitches sec. Klevmark in prolene 2/0 [1]; in 25 cases we carried out two or three series of parallel stitches in prolene $3 / 0$. The stitches crossed the total thickness of the albuginea of cavernous corps.

6 patients had evident recurvatum $\left(>60^{\circ}\right)$, wich rendered penetration almost impossible with almost impossible penetration, partial ED was present in all cases. These patients had also a DECD of cavernous corps. In these patients the ED was treated using i5PDE obtaining good results. A cycle of systematic i5PDE therapy (two/ three times week for 8 weeks) was administered to all patients before surgery.

The length of penis before grafting was between 11,5 to $14,5 \mathrm{~cm}$ $(13+/-1 \mathrm{~cm})$.

6 patients were operated on the plaque incision with partial dissection and grafting a patch obtained with thin strips of polyester fluoropolymer (Gore-Tex) (Figure 2)

The patch was 30\% larger than the exposed cavernosal area. The strips were sutured with gore-tex stitches 5-6/0 (Figure 3).

In all 46 patients we carried out extensive dissection of the dorsal plexus and the urethra (Figure 3). We performed the postectomy and patients were discharged from hospital on the day after surgery with the recommendation to avoid intercourse for 6 weeks.

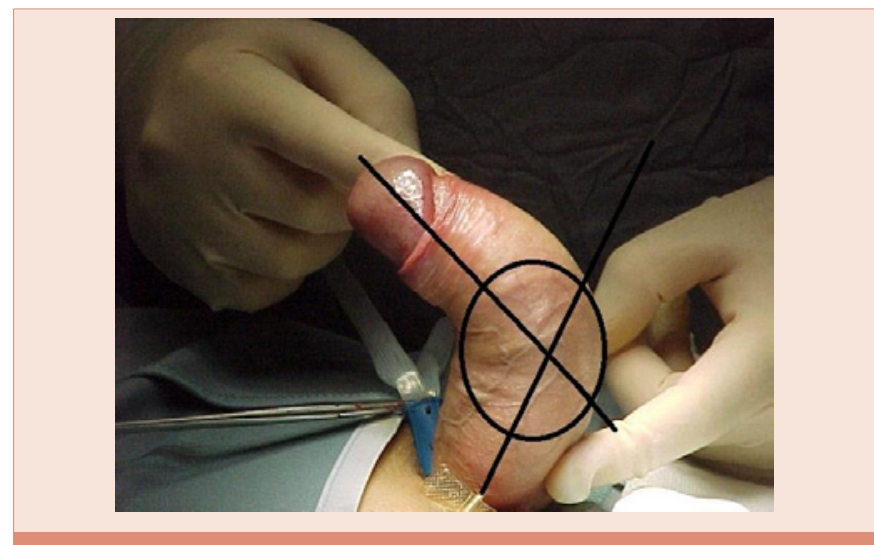

Figure 1: Photography of the erect penis, documents the importance of recurvatum.

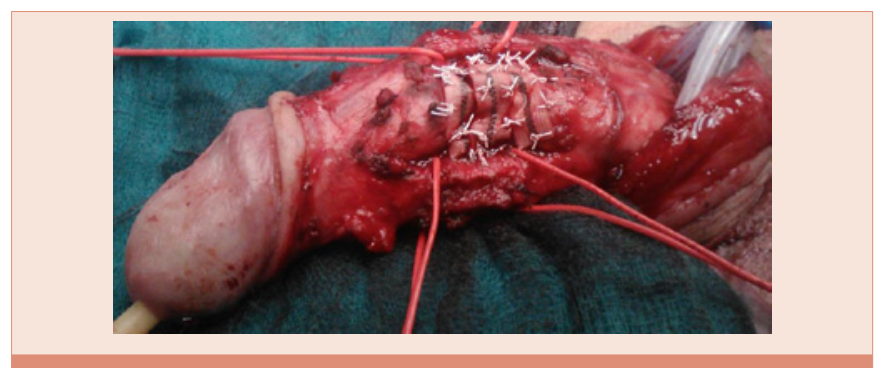

Figure 2: Partial dissection of the plaque and grafting a patch obtained with thin strips of polyester fluoropolymer sutured with gore-tex stitches $5 / 0$.

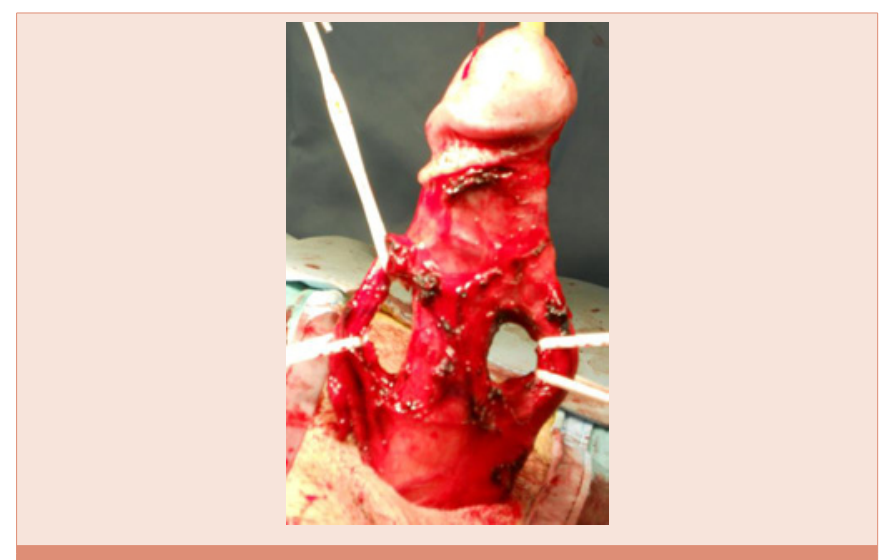

Figure 3: Extensive dissection of the dorsal plexus and the urethra.

\section{Results}

The follow-up was between 1 and 5 years. The correction of recurvatum was good.

- In patients treated with albuginea plication the penile shortening was between 0,5 and $2 \mathrm{~cm}$ (Chart 1). Patients operated according Klevmark, reported little nodulations in the sites of stitches. All these patients did not report recurrence of recurvatum. 
- In patients treated with grafting and patch we obtained an excellent correction of the recurvatum. The shortening was insignificant (Chart 2) and the patients continued to use 5PDEi.The graft has never induced clinical signs of inflammation, scar retractions or pseudo-aneurysm expansion, even in the medium term (5 years).

The surgery (albuginea plication or dissection with patch) did not have any short term complications (in particular urethral injury). Most patients suffered of glans hypoesthesia with hypoorgasmya for 3-12 weeks.

A questionnaire was administered 6-8 months after surgery to evaluate patient satisfaction.

Patients treated with albuginea plication, 87\% (35pts) were "very satisfied" or "satisfied enough" with the results of the procedure (Chart 1).

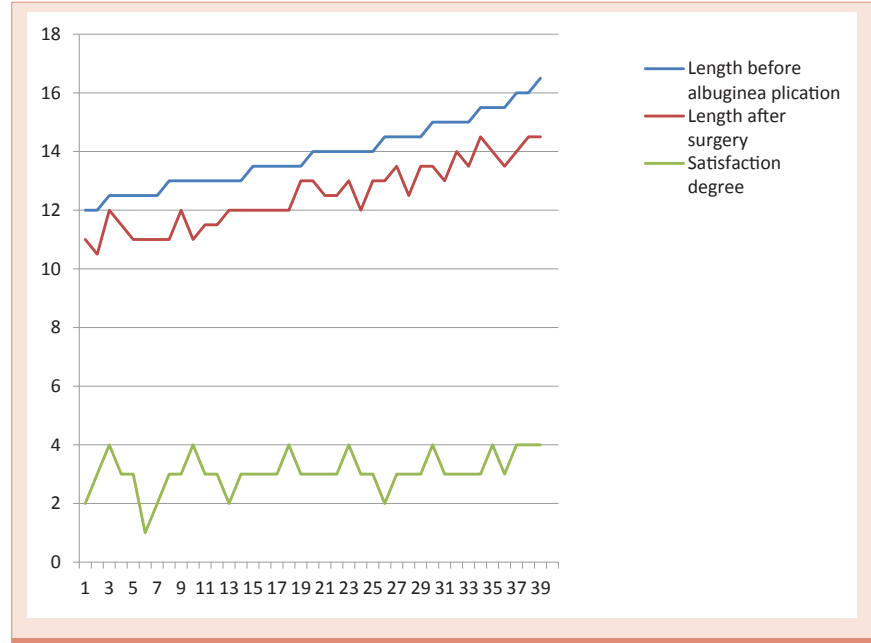

Chart 1: Length before $(12-16,5 \mathrm{~cm}-\mathrm{m} .14+/-1,2 \mathrm{~cm})$ and after $(10,5-14,5 \mathrm{~cm}-$ $\mathrm{m} .12,5+/-1 \mathrm{~cm})$. albuginea plication and satisfaction degree (m. score3).

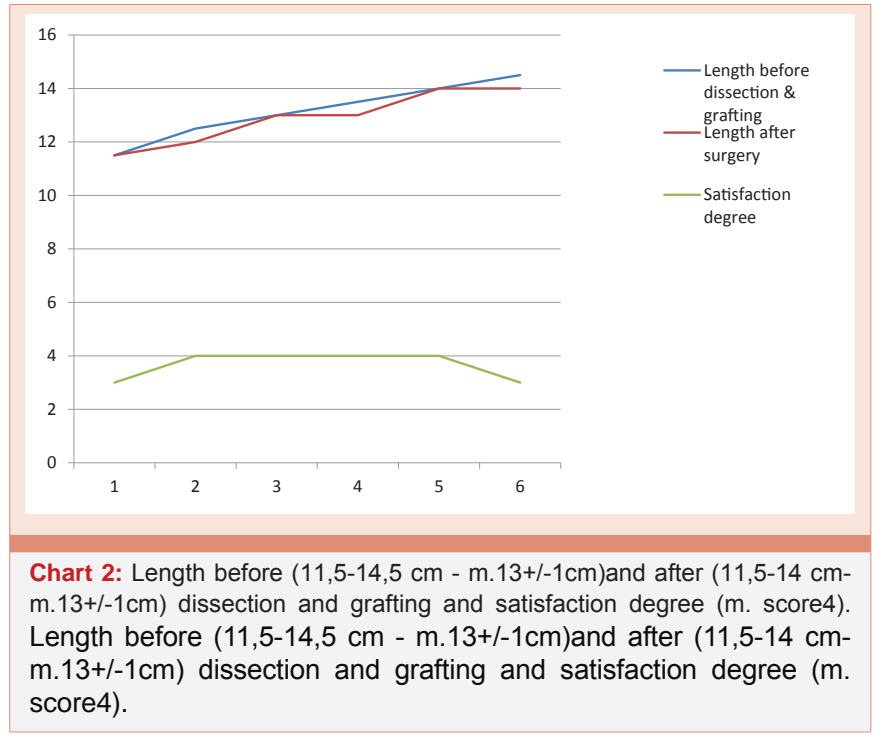

All patients treated with dissection and patch were either "very satisfied" or "satisfied enough" (Chart 2).

\section{Discussion}

The Induratio Penis Plastica was described by Francois Gigot de la Peyronie in 1743 [2]. This condition is characterized by a sclerotic lesion of the tunica albuginea of Cavernous Corps. It affects the tunica albuginea in a segmental way and, sometimes multiple nodulations may coexist in different sites of the cavernous corps. While etiology is unknown, the pathogenesis is probably related to immune mechanisms secondary to micro traumatic repeated events, triggering the macrophage proliferation. Such events would activate the development of a plaque and would be regulated by local growth factors -TG $\beta 1$-[3]. A pathogenetic explanation involving stem cells has also been proposed: these cells would differentiate in osteoblasts or fibroblasts, causing the growth of the fibrous plaque and/or calcified areas [4].

The macrophagic activation would lead to the alteration of the structure of the albuginea with reduction of the elastic fibers so that the albuginea loses its compliance: the plaque thus represents the point of in-extensibility and recurvatum and sandglass deformity are the consequence of it.

Peyronie's disease is a relatively common condition and the calculated incidence is 3-9\% [5].

While diagnosis is largely clinical, some diagnostic tools are needed in order to guide the most appropriate therapeutic strategy.

Medical therapy is proposed for light recurvatum ( $<45$ degrees) $[3,6]$, in patients without erectile dysfunction and with mild coital disorders, with a disease span of less than 12 months. In case of moderate or considerable recurvatum, surgical therapy is the standard treatment.

In these cases, it is appropriate to make an accurate assessment of the length of the penis during stretching and measuring it with a rigid ruler [7], the measure of length of the penis, before surgery is useful to inform patients that shortening of cavernous corps, is caused both by surgery and by the condition.

Is very important to verify the curvature angle with auto/eterophotography of the erect penis [6]. Peyronie's disease is associated with $\mathrm{ED}$ in $58 \%$ of cases [3], and for this reason is important evaluating erectile function taking a good patient history and assessing the response to IIEF, if necessary with DECD [6].

The aim of surgery in la Peyronie's disease is to correct penile deformity. This can be achieved either reducing length of the convex part of cavernous corps, or with a section/dissection of the plaque with grafting [3].

The first procedure was described by Nesbit and it consist in the dissection of the some ellipse of albuginea with suture of the margins or the application of some stitches to obtain the plication of the convex part of the albuginea. These could be " $U$ " in shaped not resorbable material (Prolene, Gore-tex). 
Albuginea plication is indicated for $45-60^{\circ}$ recurvatum, without Erectile Dysfunction and with mild sandglass deformity [7].

For recurvatum $>60^{\circ}$ with partial erectile dysfunction (usually erectile instability) and with sandglass deformity, it is indicated the section/dissection of the plaque with grafting $[9,10]$.

Albuginea plication offers a good correction of recurvatum with restoration of a correct coital function, but it is associated with a reduction of the corporal length between 1 and $3 \mathrm{~cm}$, in $30-90 \%$ of cases [6]. Penis length before the procedure must be noted in order to correctly inform the patient of this complication. Measuring penis length before surgery allows to accurately predict what it will be after the procedure; only a penile length $10 \mathrm{~cm}$ or more is considered compatible with a satisfactory sexual function [11].

Grafting does not cause a reduction of penile length, but in 15$45 \%$ of cases it is burdened from erectile dysfunction. Patient selection and careful information are essential [6].

The patch needs to be elastic and compliant enough to allow the expansion of the cavernous muscular tissue, but it does not expand too much so as in a pseudo-aneurysm.

The patch is not antigenic, does not induce inflammation and is relatively cheap [9].

A variety of materials have been used to produce the patches:

Authologous tissues (vein grafts, buccal mucosa, rectum muscle fascia, tunica vaginalis and crural albuginea); biologic cadaveric tissues (dura matter, pericardium, cadaveric dermis); xenografts (porcine small intestine sub-mucosa, bovine pericardium); synthetic grafts (gore-tex, Dacron) [6,9].

Synthetic grafts are preferred in guide lines, but this is controversial as inflammation and sclerosis was seen in a limited number of cases near the patch. In some cases synthetic grafts became infected or causes pseudo-aneurisms [12], nevertheless some authors reported good results with gore-tex grafting [10].

The thin strips of polyester fluoropolymer, are thin, inert and elastic enough to consent the corporal expansion; it is very important to make a graft $30 \%$ larger of the dissected area. In this way the patch is not stretched excessively during erection and the "giving in" is then prevented.

During the surgical procedure it is very important to dissect the cavernous corps, the dorsal plexus and the urethra [13], in this way it is possible to place the plicature stitches over all the albuginea thickness and incising and partially dissecting the IPP plaque. The use of Gore-Tex devices in plaque surgery started in the nineties, taking as a model its use in vascular surgery. Their use in Peyronie's Disease is advised by the guidelines enforced by urological societies [6]. Unfortunately, there are no studies proving the biocompatibily of Gore Tex in the andrology setting. In fact, a large study carried out in vascular surgery (298 patients studied after a median of 500 days the device was inserted) documented cellular and collage micropore infiltration. Anyway, this was poor and only an irregular layer of organized fibrin was documented [14]. The presence of cellular infiltration and periprosthetic fibrin deposition was shown in rabbits with implanted gore-tex iliac endovascular grafts: this inflammatory reaction did not alter the width of the intravascular lumen [15]. In another experimental study, it was demonstrated that the injection of a solution containing gore-tex in muscular tissue of rats triggered an inflammatory reaction creating a pseudo-capsule around the injected material, with only one case of granuloma formation [16]. In conclusion, while in experimental studies Gore Tex devices can cause inflammation characterised by cellular micropore infiltration, this does not appear to be clinically relevant, as confirmed by our study as well.

\section{Conclusion}

Our study shows that albuginea placation is a useful technique, giving a good and lasting results, when correctly indicated.

In case of important recurvatum and penile deformity with ED and difficult or impossible penetration, it is indicated the incision/ dissection of plaque with grafting. In our experience the synthetic tissues -strips of polyester fluropolymer- if well shaped, offer good results with no severe complications, in the short or in the long term.

\section{References}

1. Klevmark B, Andersen M, Schultz A, Talseth T (1994) Congenital and acquired curvature of the penis treated surgically by placation of the tunica albuginea. Br J Urol 74: 501-506.

2. Francois Gigot de la Peyronie (1743) Memoire sur quelques obstacles qui s'opposent à l'ejaculation naturelle de la semence Memoires de l'Academie Royale de Ghirurgie 425.

3. Ralph D, Gonzalez-Cadavid N, Mirone V, Perovic S, Sohn M, et al. (2010) The management of Peyronie's disease: evidence-based 2010 Guidelines J Sex Med 7: 2359-2374.

4. Vernet D, Nolazco G, Cantini I, Magee TR, Qian A, et al. (2005) Ebidence that osteogenic progenitor cells in the human tunica albuginea may originate from stem cells: implications for peyronie's disease. Biol Reprod 73: 199-210.

5. Kumar B, Narang T, Gupta S, Gulati M (2006) A clinico-aetiological and ultrasonographic study of Peyronie's disease. Sex Health 3:113-118.

6. Hatzimouratidis K, Eardley I, Giuliano F, Hatzichristou D, Moncada I, et al. (2012) EAU Guidelines on Penile Curvature. Eur Urol 62: 543-552.

7. Levine LA, larsen SM (2013) Surgery for Peyronie's disease. Asian J Urol 15 27-34.

8. Rosen R, Catania J, Lue T, Althof S, Henne J, et al. (2008) Impact of Peyronie's disease on sexual and psychosocial functioning. Qualitative findings in patients and controls. J Sex Med 5: 1977-1984.

9. Lentz AC, Carson CC 3rd (2009) Peyronie's surgery: graft choices and outcomes Current. Urology Reports 10: 460-467.

10. Bokarica P, Parazajder J, Mazuran B, Gilja I (2005) Surgical treatment of Peyronie's disease based on penile length and degree of curvature. Int $\mathrm{J}$ Impot Res 17: 170-174.

11. Chevallier D, Haertig A, Faix A, Droupy S (2013) Cosmetic surgery of the male genitalia. Prog Urol 23: 685-695.

12. Kadioglu A, Sanli O, Akman T, Ersay A, Guven S, et al. (2007) Graft materials in Peyronie's disease surgery: a comprehensive review. J Sex Med 4: 581595.

13. Passavanti G, Bragaglia A, Gnech M, Nucciotti R, Spinosa E, et al. (2011) 
The albuginea plication for the recurvatum penis secondary in Peyronie's disease (IPP): a traditional surgical procedure. J Sex Med 8: 444.

14. Guidoin R, Chakfé N, Maurel S, How T, Batt M, et al. (1993) Expanded polytetrafluoroethylene arterial prostheses in humans:histopathological study of 298 surgically excised grafts Biomaterials 14: 678-693.

15. Unverdorben M, Spielberger A, Schywalsky M, Labahn D, Schneider M, et al.
(2001) First reuslts in animal model on stents sheated with plytetrafuorethylene membrane. RoFo 173: 836-841.

16. Aydin O, Sengor GA, Gurbuz Y, Mola F (2007) Histopathologic study of minced and injected expanded polytetrafluoroethylene (Gore-Tex) in quadriceps muscle of rats for injection laryngoplasty. Auris Nasus Lanrynx 34: $333-338$

Copyright: (c) 2015 Passavanti G, et al. This is an open-access article distributed under the terms of the Creative Commons Attribution License, which permits unrestricted use, distribution, and reproduction in any medium, provided the original author and source are credited.

Citation: Passavanti G, Aloisi AM (2015) Surgery in Peyronie's Disease: Our Experiences with Tunica Plication, Plaque Incision and Grafting with Gore- 